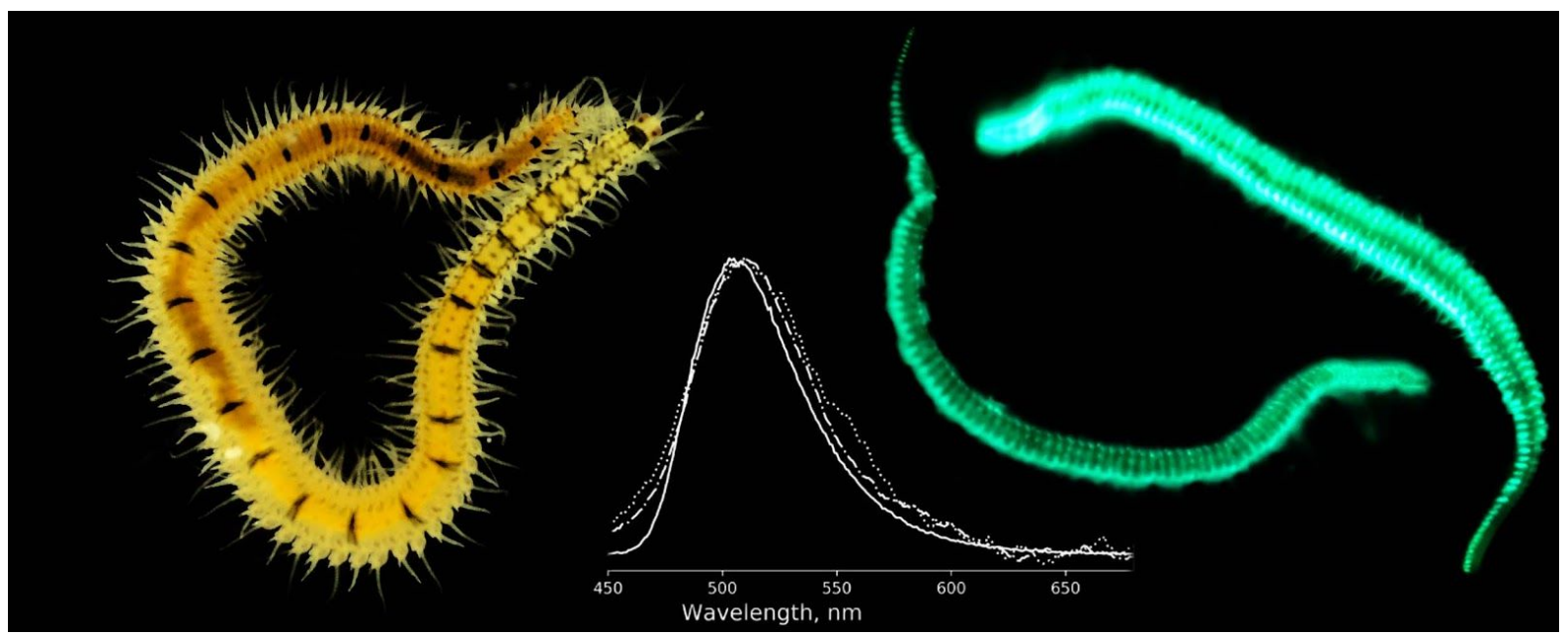

Title:

\title{
Luciferase of the Japanese syllid polychaete Odontosyllis umdecimdonta
}

\section{Authors:}

Darrin T. Schultz ${ }^{1 *}$, Alexey A. Kotlobay ${ }^{2 *}$, Rustam Ziganshin², Artyom Bannikov ${ }^{2,3}$, Nadezhda M. Markina ${ }^{2}$, Tatiana V. Chepurnyh ${ }^{2}$, Ekaterina S. Shakhova ${ }^{2}$, Ksenia Palkina $^{2}$, Steven H.D. Haddock ${ }^{4,5}$, Ilia V. Yampolsky, ${ }^{2,6, \#, ~ Y u i c h i ~ O b a ~}{ }^{7, \#}$

\section{Affilations:}

1 Department of Biomolecular Engineering and Bioinformatics, University of California Santa Cruz, 1156 High Street, Santa Cruz, California 95064, United States 2 Shemyakin-Ovchinnikov Institute of Bioorganic Chemistry of the Russian Academy of Sciences, Miklukho-Maklaya, 16/10, Moscow, Russian Federation 117997

${ }^{3}$ Planta LLC, Bolshoi Boulevard, 42 Str 1, Office 335; Moscow, Russia 121205

${ }^{4}$ Monterey Bay Aquarium Research Institute, 7700 Sandholdt Road, Moss Landing, California 95039, United States

5 Department of Ecology and Evolutionary Biology, University of California Santa Cruz, 1156 High Street, Santa Cruz, California 95064, United States

${ }^{6}$ Pirogov Russian National Research Medical University, Ostrovitianova 1, Moscow 117997, Russia

${ }^{7}$ Department of Environmental Biology, Chubu University, Kasugai 487-8501, Japan

* - These authors contributed equally to this work.

\# - Corresponding author. Correspondence and requests for materials should be addressed to ivyamp@ibch.ru and yoba@isc.chubu.ac.jp 


\section{Abstract}

Odontosyllis undecimdonta is a marine syllid polychaete that produces bright internal and exuded bioluminescence. Despite over fifty years of biochemical investigation into Odontosyllis bioluminescence, the light-emitting small molecule substrate and catalyzing luciferase protein have remained a mystery. Here we describe the discovery of a bioluminescent protein fraction from $O$. undecimdonta, the identification of the luciferase using peptide and RNA sequencing, and the in vitro reconstruction of the bioluminescence reaction using highly purified $O$. undecimdonta luciferin and recombinant luciferase. Lastly, we found no identifiably homologous proteins in publicly available datasets. This suggests that the syllid polychaetes contain an evolutionarily unique luciferase among all characterized luminous taxa.

\section{Keywords}

bioluminescence, luciferase, luciferin, Odontosyllis, Oxford Nanopore, RNA-seq

\section{Highlights}

- The polychaete $O$. undecimdonta uses a luciferin-luciferase bioluminescence system

- O. undecimdonta bioluminescence does not require additional cofactors

- The luciferase of the Japanese fireworm is 329 amino acids long

- Recombinant luciferase is not secreted when expressed in human cells

- Exogenous luciferin does not seem to penetrate cell membranes- only lysate luminesces

- The luciferase transcript is supported by full-length cDNA reads with 5' and 3' UTR 


\section{Introduction}

Odontosyllis is a widely distributed genus of marine syllid polychaete worms that are noted for their striking bioluminescent courtship displays [1-5]. The bioluminescence $(\mathrm{BL})$ of Odontosyllis is a luciferin-luciferase system [6], but the structure of the luciferin and the luciferase protein remain unknown despite several biochemical studies following the first in 1931 by Harvey [6-11]. More broadly, to date the enzyme sequences and luciferin structures remain a mystery for all polychaete species in the thirteen families containing luminous species [12].

Previous studies of the Odontosyllis bioluminescence system generated conflicting results regarding whether the system is a soluble oxygen-dependent luciferin-luciferase reaction [8,9], or is a photoprotein system in which the light-emitting small molecule substrate is covalently bound to the enzyme [11]. The above studies used a different Odontosyllis species, and the different colors of aqueous extracts identified from those species make it unclear whether there are multiple bioluminescent chemistries within Odontosyllis. However, both species have the same behavior of secreting luminescence during mating $[1,4]$, so both species presumably share a homologous bioluminescent system.

Odontosyllis undecimdonta is a species found in Toyama Bay, Japan which engages in bioluminescent surface courtship displays around the first new moon in October [13]. Recently a protein-coding sequence from $O$. undecimdonta was patented that produces a recombinant protein with luminescence activity similar to that of crude worm extract mixed with crude luciferin isolate (WO2017155036A1). Here, we describe the identification, cloning, and characterization of the 0 . undecimdonta luciferase. In addition, our results suggest that the $O$. undecimdonta luminescence system is a luciferase-luciferin type without requisite cofactors, despite reports of magnesium ions as a necessary cofactor [14]. 


\section{Materials and Methods}

\subsection{Specimen Collection}

Professor S. Inoue provided lyophilized O. undecimdonta worms collected in 1993 to develop the protein purification strategy [15]. The final specimens used in this study for protein purification, MS transcript identification, and nucleic acid purification were collected on October 06, 2016 in Toyama Prefecture Japan, Namerikawa City. At dusk, Odontosyllis worms were attracted to a handheld light at the surface and collected with a hand dip net. Worms were individually preserved in Invitrogen RNAlater or lyophilized for later analysis.

\subsubsection{DNA and RNA isolation}

Methods for DNA and RNA isolation, as well as construction of the RNA-seq and genomic DNA libraries are as described in the Supplementary Information. Briefly, the O. undecimdonta transcriptome was assembled using 32,457,166 Illumina 2x150 read pairs and 343,752 Oxford Nanopore long reads using the Trinity assembler [16]. DNA from a single $O$. undecimdonta specimen was used to prepare both a 10X Genomics chromium library [17] and a PCR-free library. All sequencing reads are available to download from the European Nucleotide Archive under project PRJEB26709. Individual luciferase transcripts are available at NCBI accession numbers MH350412 and MH350413.

\subsection{Protein extraction from biomaterial}

Five $\mathrm{ml}$ of phosphate buffer ( $5 \mathrm{mM}$ sodium phosphate buffer, $\mathrm{pH}$ 7.4) was added to $150 \mathrm{mg}$ of lyophilized worms. Then this mixture was dropped in to the liquid nitrogen, using a $1 \mathrm{ml}$ pipette, to create small drops of frozen material. These small ice drops were ground in a mortar. Frozen powder was added to $10 \mathrm{ml}$ of phosphate buffer ( $5 \mathrm{mM}$ sodium phosphate buffer, $\mathrm{pH}$ 7.4) and incubated $40 \mathrm{~min}$ on an ice bath with stirring. After incubation this solution was centrifuged at $40000 \mathrm{~g}$ 
$\left(4^{\circ} \mathrm{C}\right)$ for $40 \mathrm{~min}$. The supernatant, containing luciferase, was then collected and used for further purification by anion exchange chromatography.

\subsection{Protein purification}

\subsubsection{Anion exchange chromatography of water extract.}

An extract of $O$. undecimdonta was applied to a DEAE Sepharose (GE Healthcare, Uppsala, Sweden) HiTrap Fast Flow column $(1.6 \times 2.5 \mathrm{~cm})$, equilibrated and washed with $5 \mathrm{mM}$ sodium phosphate buffer, $\mathrm{pH} 7.4$ at rate of $5 \mathrm{ml} / \mathrm{min}$. The elution was done by linear $\mathrm{NaCl}$ gradient from 0 to $0.4 \mathrm{M}(80 \mathrm{ml})$ and $5 \mathrm{ml}$ fractions were collected. To minimize bioluminescent reactions, the solvent, fractions and column were maintained at $4^{\circ} \mathrm{C}$. Automatic fraction collection and solvent application was controlled with an Akta Prime chromatography system (GE Healthcare, Uppsala, Sweden). After elution, fractions containing luciferase and luciferin were detected by pairwise mixing all possible fraction combinations. The reaction was monitored with a custom-made luminometer Oberon-K (Krasnoyarsk, Russia).

\subsubsection{Ultrafiltration and concentration.}

To discard additional proteins from the luciferase-containing fractions the ultrafiltration procedure was used. First, the active fraction was filtered on a $50 \mathrm{kDa}$ Amicon $^{\circledR}$ Ultra centrifugal filter unit (Merck Millipore, Germany). BL activity was measured for the concentrated retentate and the permeate. We found that only the permeate was bioluminescent. The bioluminescent permeate was then concentrated on 30 kDa Amicon ${ }^{\circledR}$ Ultra centrifugal filter unit (Merck Millipore, Germany). The resulting retentate possessed BL activity while the permeate did not. Thus this concentrated luciferase sample was used for size exclusion chromatography.

\subsubsection{Size exclusion chromatography.}

The bioluminescent retentate from ultrafiltration was applied to a Superdex 200 column (Phenomenex, USA) on a Shimadzu chromatography system (Shimadzu, 
Japan). The loaded column was washed with $5 \mathrm{mM}$ sodium phosphate buffer, 150 $\mathrm{mM} \mathrm{NaCl}, \mathrm{pH} 7.4$ at rate of $0.4 \mathrm{ml} / \mathrm{min}$. During separation $0.5 \mathrm{ml}$ fractions were collected. The solvent, fractions, and column were maintained at $4^{\circ} \mathrm{C}$. BL-active fractions were used in the subsequent gel electrophoresis experiments.

\subsection{Denaturing polyacrylamide gel electrophoresis and amino acid} sequence analysis.

SDS-PAGE of the BL-active fractions was performed using a 10-25\% gradient gel according to [18]. Gel staining was done according to the silver staining protocol from [19], or using a standard Coomassie G250 staining protocol. Protein bands were excised from the gel and subjected to in-gel trypsinolysis [20]. LC-MS was performed on the Ultimate 3000 Nano LC System (Thermo Fisher Scientific), connected to a $Q$ Exactive HF mass spectrometer (Thermo Fisher Scientific). For data analysis, Mascot software (Matrix Science) with the $O$. undecimdonta transcriptome as a reference was used.

\subsubsection{Molecular cloning}

Four Odontosyllis luciferase candidate genes were codon-optimized for expression in mammalian cells, domesticated for compatibility with MoClo assembly [21] and ordered from a commercial supplier (Twist Biosciences, USA) as linear dsDNA fragments. Molecular cloning is described in detail in the Supplementary Materials.

\subsubsection{Mammalian cell culture}

HEK293NT cells were grown under standard conditions and transfected with FuGene 6 reagent (Promega, Fitchburg, WI, USA) in accordance to the manufacturer's protocol. For more details see Supplementary materials.

\subsection{In vitro bioluminescence assay}

The reaction was monitored with a custom-made luminometer Oberon-K (Krasnoyarsk, Russia) at room temperature. For each measurement $100 \mu \mathrm{l}$ of reaction mix $(10 \mathrm{mM}$ sodium phosphate buffer, $150 \mathrm{mM} \mathrm{NaCl}, \mathrm{pH} 7.4,2 \mu \mathrm{l}$ of 
luciferase fraction, $2 \mu \mathrm{l}$ of highly purified luciferin [22] were used. In experiments with mammalian cells lysates, the same amount of cells was used for each clone in each bioluminescence analysis to make results comparable.

The involvement of additional cofactors in the O. undecimdonta bioluminescence reaction was tested using an in vitro assay with only purified luciferase and highly purified luciferin. Since previous studies suggest the involvement of $\mathrm{Mg}^{2+}$ in the Odontosyllis bioluminescence reaction (optimum conc is $30 \mathrm{mM}$; [14]), we also testing the in vitro bioluminescence assay with $30 \mathrm{mM}-60 \mathrm{mM} \mathrm{Mg}{ }^{2+}$ with cell lysate.

\subsection{Protein structure and homology analysis}

HMMER and the BLAST suite were used to predict structural domains and interspecies homology of transcripts that produced bioluminescence [23-25]. We also used Phobius and SignalP to detect signal peptides and transmembrane domains of the same transcripts [26,27]. Lastly, we used the I-TASSER server for structural prediction [28]. See the supplementary materials for a detailed description of the search for homologous sequences.

\section{Results}

The isolation and purification of $O$. undecimdonta luciferase required ion exchange chromatography, size exclusion chromatography, and ultrafiltration. (Fig. 1). The presence of luciferase in samples was controlled by an in vitro BL assay for all stages of purification. Several bands that corresponded to BL activity in the size exclusion chromatography fractions were identified by polyacrylamide gel electrophoresis (Fig. 1C). These bands were excised from gel and were identified by LC-MS.

The transcriptome assembled with Illumina paired-end reads and ONT 2D reads extracted with poretools "fwd" parameter yielded 256,027 transcripts and a median transcript length of 737 base pairs. Four transcripts were identified as potential luciferases (Fig. 2) based on coverage and quantity of MS matches. Three 
long transcripts c9g1i2 (990 bp), c9g1i3 (993 bp), c9g1i6 (990 bp) had c-terminal amino acid variation. Transcript c9g1i5 (711 bp) was homologous to the aforementioned three transcripts but lacked $118 \mathrm{n}$-terminal amino acids. These four transcripts were verified by presence of two ONT whole-cDNA reads that spanned from the 5' UTR to the 3'UTR. Non-spliced mapping of an Illumina paired-end polyA RNA-seq library also confirmed that the longest of the four transcripts were expressed. The BLOSUM-alignment for the protein products of these four transcripts were identical at $92 \%$ of sites.

All four candidate DNA sequences were synthesized as linear dsDNA fragments and cloned using MoClo technology. Then, mammalian cells were transfected by resulting constructs. Mammalian cell culture lysate from two of the above four candidates produced bioluminescence when assayed with purified luciferin (c9g1i2 and c9g1i6) (Fig. 3A). The bioluminescence spectra of positive clones were similar to that of native O. undecimdonta worms (Fig 3B). However, cell culture lysate from expressed transcripts c9g1i3 and c9g1i5 were not luminous. None of the non-lysed cell cultures produced luminescence when purified luciferin was applied.

The protein product of c9g1i2 is 329 amino acids long. The signal peptide prediction software Phobius had a posterior probability of 1 that the first 21 c-terminal peptides are a signal peptide. The SignalP service has a probability of 0.28 that the first 21 amino acids are signal peptides. The only HMMER and PHMMER results for this protein product were an insignificant match $(E-v a l u e=0.8)$ to a prokaryotic protein involved in mRNA production. I-TASSER protein structure and function prediction results found that nine of the top ten structural homologs to the protein product of $\mathrm{c} 9 \mathrm{~g} 1 \mathrm{i} 2$ were adenosine deaminase/hydrolases. A tblastn search with the $c 9 g 1 \mathrm{i} 2$ protein product only found an insignificant match (E-value = 3.1) to a predicted gerbil transcription factor ( sequence XM_021634012.1). A blastn search returned no significant matches. Blast searches against the assembled transcriptomes of publicly available polychaete RNA-seq read data also yielded no significant matches (SI results).

The mixture of purified 0 . undecimdonta luciferase and luciferin in TBS (50 $\mathrm{mM}$ Tris- $\mathrm{HCl}, 150 \mathrm{mM} \mathrm{NaCl}, \mathrm{pH}$ 8.0) was luminescent, even in the absence of $\mathrm{Mg}^{2+}$ 
ions. Increasing the $\mathrm{Mg}^{2+}$ concentration in the reaction buffer of recombinant luciferase cell lysate did not affect the yield of the bioluminescence reaction (data not shown).

\section{Discussion}

Given our lack of fresh specimens we opted to extract and purify the Odontosyllis luciferase directly from the lyophilized worms and successfully identified the luciferase gene using classic protein purification, luciferin purification, and recent whole-cDNA sequencing techniques. We then reconstructed native Odontosyllis bioluminescence in vitro using purified protein and highly purified luciferin [22] with no additional cofactors. Lastly, we verified the identity of the Odontosyllis luciferase gene by showing that recombinant protein and purified luciferin in cell-lysate is luminous, in which the luminescence spectra ( $\lambda \max$, near $510 \mathrm{~nm})$ matches that of the Odontosyllis in vivo luminescence.

It is notable that using purified components in studying bioluminescence reactions is important to verify that off-target reactions are not the source of luminescence and to avoid erroneous interpretation of the results [14]. Given that the protein and luciferin purification products were luminous and that luminescence of recombinant luciferase cell lysates were not enhanced with $\mathrm{Mg}^{2+}$ the $\mathrm{O}$. undecimdonta luciferase-luciferin reaction does not appear to require additional cofactors. It is also important to note that the recombinant protein is not secreted by eukaryotic cells and that the luminescence reaction only occurs when cells containing the recombinant luciferase are lysed. This suggests that the highly purified $O$. undecimdonta luciferin is not membrane-permeable, thus limiting the potential for applications of this luciferase in optogenetics or other cellular expression-based technology.

While the bioluminescence emitted during mating is well-characterized in Odontosyllis spp., the luciferase structure and the mechanism of the luciferin-luciferase reaction remains unclear. Despite this uncertainty, protein ortology searches using BLAST and HMMER show that syllid luciferase is unique both among sequenced polychaetes and other sequenced organisms in public 
databases. The lack of evidence for a conserved protein in the transcriptomes of other luminous polychaetes leaves open the theory that bioluminescence evolved more than three times in the annelids. In this conservative estimate, we only include the evolution of two unique bioluminescent systems for which either the structure of the luciferin, luciferase, or both have been determined (earthworms [29] and Odontosyllis), plus at least one event for other annelids with uncharacterized bioluminescent systems.

Given that the structure of other polychaete luciferins is still unknown, this leaves the question of polychaete bioluminescence unanswered. Identification of the 0 . umdecimdonta luciferase sequence is the most important step to further characterization of this worm's bioluminescent system and the screening of other purified polychaete luciferins for cross-reactivity.

\section{Acknowledgements}

We thank late Professor Shoji Inoue and Dr. Hisae Kakoi (Meijo University, Japan) for providing lyophilized Odontosyllis specimens, and Uozu Aquarium (Toyama, Japan) for help collecting Odontosyllis specimens.

\section{Funding}

This work was supported by the Russian Science Foundation grant 14-50-00131, the David and Lucile Packard Foundation, and the Monterey Bay Aquarium Research Institute. DTS was supported by the NSF GRFP DGE 1339067. 


\section{Figure/Table Legends}

Fig. 1. Purification and isolation of O.undecimdonta luciferase. A - Chromatographic profile of water extract from lyophilized $\mathrm{O}$. undecimdonta worms anion-exchange chromatography on DEAE Sepharose HiTrap Fast Flow column. Solid line - $280 \mathrm{~nm}$ absorption signal. Activity profile of Odontosyllis luciferase shown as dashed line. B - Size exclusion chromatographic profile of anion-exchange chromatography concentrated luciferase fractions on Superdex 200 column. Solid line - $280 \mathrm{~nm}$ absorption signal. Activity profile of $O$. undecimdonta luciferase shown as dashed line. C - SDS-PAGE analysis of size exclusion chromatography fractions. Lanes: 1 PageRuler protein ladder (Thermo Scientific), lanes from 2 to 8 - size exclusion chromatography fractions 13-19 respectively. Arrows shows protein bands excised from gel and analyzed by LC-MS. D - Normalized luminescence activity of size exclusion chromatography fractions, used for SDS-PAGE analysis.

Fig. 2. Supporting evidence for transcript models aligned to the c9g1i2 transcript, including 5' and 3' UTR. The Peptide Matches track shows unique peptide hits to any of the four transcript models that match by DNA and amino acid sequence similarity. All transcript models except c9g1i5 share the same structure, whereas c9g1i5 lacks $93 \mathrm{~N}$-terminal amino acids. The ONT cDNA Reads track shows individual Oxford Nanopore 2D cDNA reads that align to the c9g1i2 transcript. Three reads span the complete 5' UTR, transcript, and 3' UTR of the long isoforms (c9g1i2, c9g1i3, c9g1i6), and four additional reads support the 5' UTR of the long isoforms. The RNA-seq coverage track supports the 5' and 3' UTR of the long isoforms, despite the predictable 3' bias inherent to polyA-selecting library preparation techniques.

Fig. 3. O. umdecimdonta luciferase properties. A - Kinetics of bioluminescence of transfected by $O$. umdecimdonta luciferase genes candidates HEK293NT cells lysates (log scale). For HEK293NT cells lysates, transfected by plasmids with c9g1i6, c9g1i2 , c9g1i5, c9g1i3 - circle, triangle, square and asterisks markers were used respectively, and rhombus marker for GFP control. B - Normalized bioluminescence spectra of natural O.undecimdonta luciferase (solid line), c9g1i6 recombinant protein (dash-dotted line) and c9g1i2 recombinant protein (dotted line). The spectral maxima are near $510 \mathrm{~nm}$.

Fig. 4. The amino acid alignment of the four luciferase transcripts. Black boxes surrounding the alignment indicate regions to which there were exact MS peptide matches. The four transcripts are on average $94 \%$ similar to one another. Transcript c9g1i5 lacks $93 \mathrm{~N}$-terminal amino acids. All transcripts have a highly variable $\mathrm{C}$-terminus. 


\section{Figures/Tables}

Fig. 1
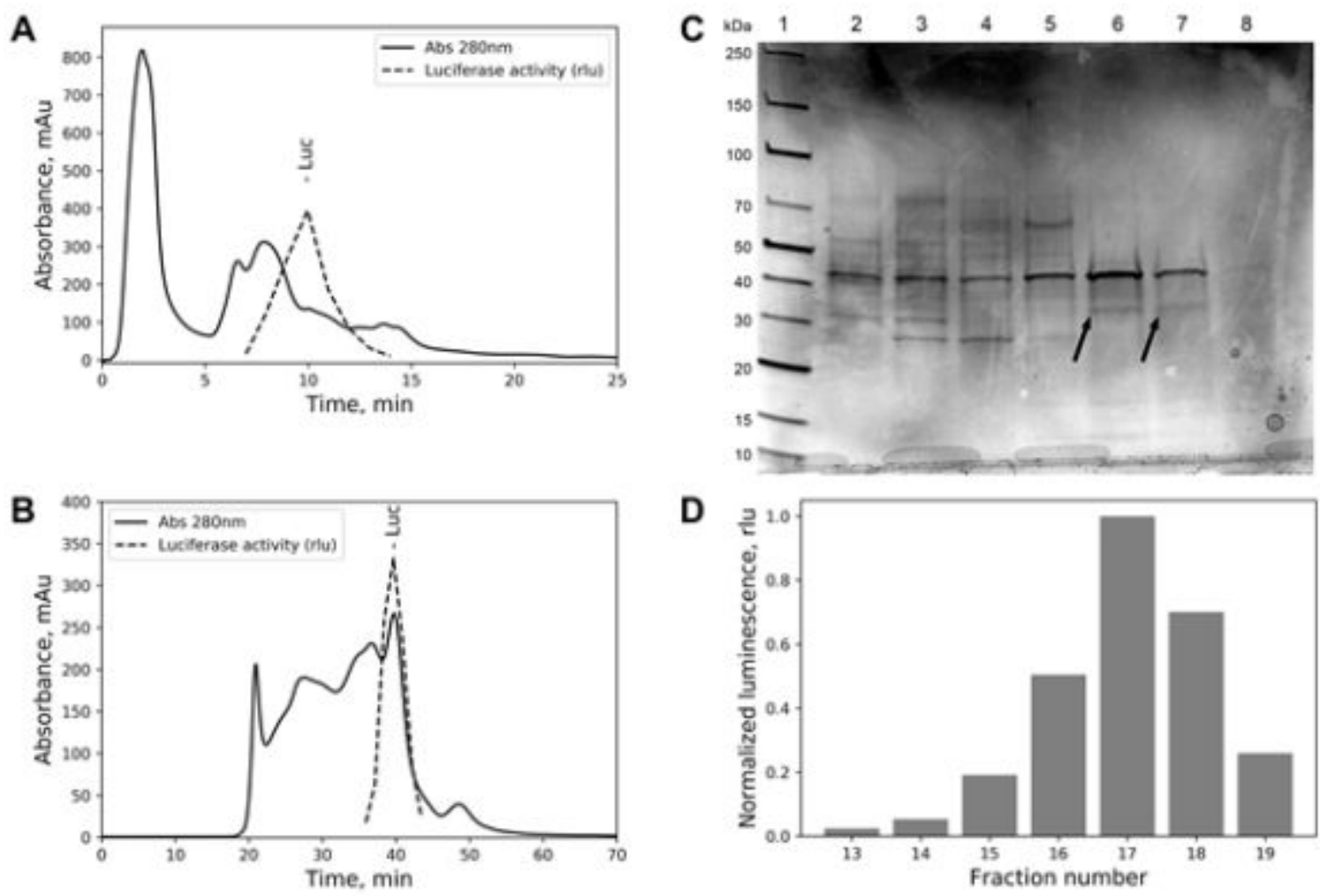

Fig. 2

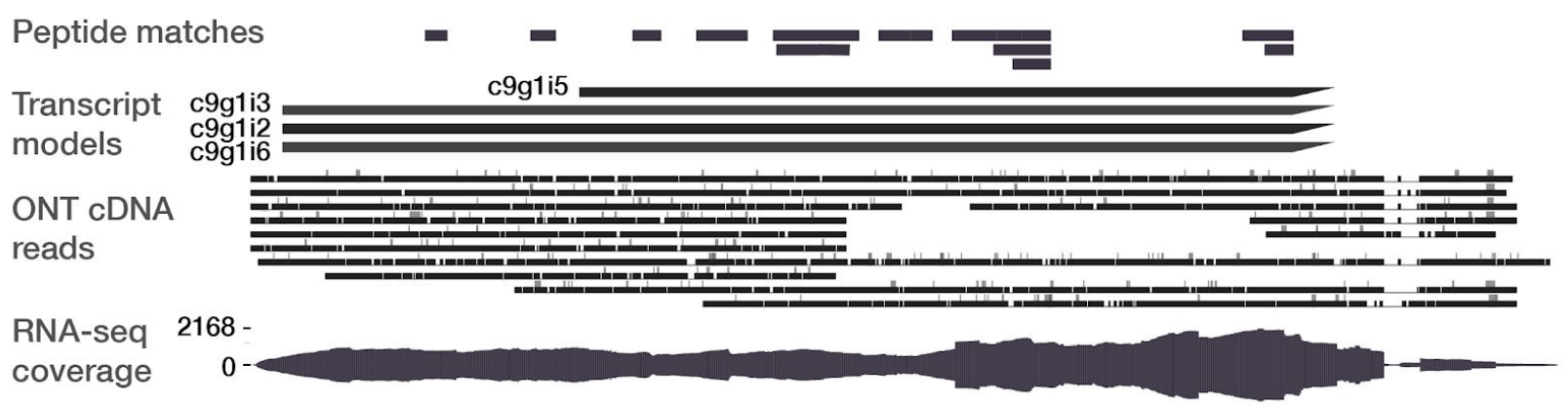


bioRxiv preprint doi: https://doi.org/10.1101/329631; this version posted May 24, 2018. The copyright holder for this preprint (which was not certified by peer review) is the author/funder, who has granted bioRxiv a license to display the preprint in perpetuity. It is made available under aCC-BY 4.0 International license.

Fig. 3

\section{A}

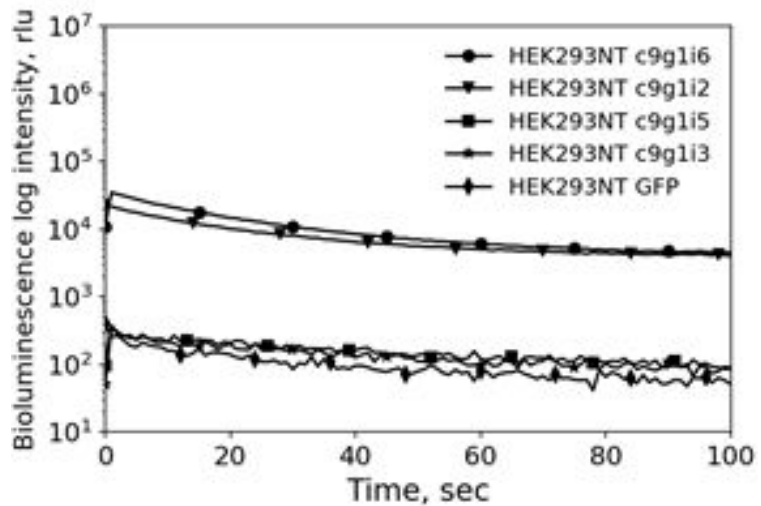

B

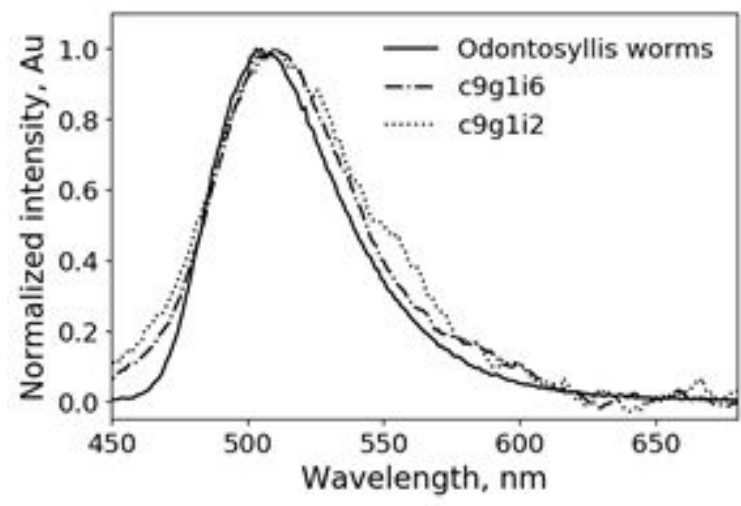

Fig. 4

1. c9_g1_is

2. c9_g1_i2

3. $c 9$ g1_i6

4. $c 9$ g1_i3

1. c9_g1_i5

2. $\mathrm{C} 9 \mathrm{~g} 1 \mathrm{i} 2$

3. $c 9-g 1$ i6

4. c9_g1_i3

1. c9_g1_i5

2. $\mathrm{c} 9 \mathrm{~g} 1$ i 2

3. $c 9-g 1$ i6

4. $c 9$ g1_i3

1. c9_g1_i5

2. $c 9$ g1 i 2

3. $c 9 . g 1$ i6

4. $c 9$ g1_i3

1. c9_g1_i5

2. $c 9 . g 1$ i 2

3. c9_g1_i6

4. $c 9$ g1_i3

110

10

30

40

50

60

MKLALLLSIGCCLVAVNFALRATI IRCLRKTRSWSEIDCTPHODKLYEDFDRIWAGDYLSVFAEWI MKLALLLSI GCCLVAVNFALRATI IRCLRKTRSWSEIDCTPHODKLYEDFDRIWAGDYLSVFAEWI MKLALLLSIGCCLVAVNFALRATIIRCLRKTRSWSEIDCTPHQDKLYDFDRIWAGDYLSVFAEWL 70 80 90 100

110 20130 MVDYMNİHGYAPFCMER'SVEDWVNARF́'TTRCKVRTDŔSL DNPIPPEWSEORLATYCIERECHTNOAMVDYMNIHGYAPFCMERSVEDWVNARFWTRCKVRTDRSL DNPIPPEWSEORLATYCIERECHTNOAMVDYMNIHGYAPFCMERSVEDWVNARFWTRCKVRTDRSI DNP I P EWSEQRLATYCIERECHTNQAMVDYMNI HGYAPFCMERSVEDWVNARFWTRCKVRTDRSI 140 160 170 180 190

ELAPEEYÁTYFCYKVFRV́ DPKIACPGḾDVILSPN KLTVQQMMQNKEIRGVVADKSEQ́WWVGIMRE ELAPEEYATYFCYKVFRVQDPKIACPSMDVILSPNKLTVQOMMQNKEIRGVVADKSEOWWVGIMRE ELAPEEYATYFCYKVFRVQDPKIACPSMDVILSPNKLTVQQMMQNKEIRGVVEDRSEOWWVGLMRE ELAPEEYATYFCYKVFRVQDP KIACPSDVILSPNKLTVQQMMQNKEIRGVVEDRSEQWWVGLMRE $\begin{array}{lllllll}200 & 210 & 220 & 230 & 240 & 250 & 260\end{array}$

IMHLSRDLNGVROFHYGWIINTATOKNVVPLWSRYQGPTVPV́RRDMPRIINÁMSNGGGNITLGDIR IMHLSKDLNGVRQFHYGWIINTATQKNVVPLWSRYQGPTVPVRRDMPRIINAMSNGGGNITLGDIR ISHLSKDLNGVKOFHYGWIINTATOKNVVPLWSRYOGPTIPVRRDMPRIINAMSNGRGNITLGDIR ISHLSRDLNGVKQFHYGWIINTATQKNVVPLWSRYQPTIPVRRDMPRIINAISNGGGNITLGDFR $\begin{array}{llllll}270 & 280 & 290 & 300 & 310 & 320\end{array}$ NFH C S A P D SVAVICPEFGF L S S PAETIVMVPVNGLILMGMTQSADGVPFVRSALFVEVYNLQQ NFHCSADPDSVAVICPEFGFLSYSPAETIVMVLVNGLILMGMTOSADGVPFVKSALFAEMYNLQQ NFHCSADPDSVAVICPEFGFLSYSPAETIVMVPVNGLILMGMTOSADGVPFVKSALFVEVYNLQQ NFECSPDPD SVALICPEFGF L S DP PETIVMVPVNELILMGMTQSADGVPLLKSALLAEIDVISQA 


\section{References}

[1] T.W. Galloway, P.S. Welch, Studies on a phosphorescent Bermudan annelid, Odontosyllis enopla Verrill, Trans. Am. Microsc. Soc. (1911). http://www.jstor.org/stable/3221049.

[2] E. Berkeley, Swarming of Odontosyllis phosphorea, Moore, and of other Polychæta near Nanaimo, B.C, Nature. 136 (1935) 1029.

[3] G.R. Gaston, J. Hall, Lunar periodicity and bioluminescence of swarming Odontosyllis luminosa (Polychaeta: Syllidae) in Belize, Gulf Caribb. Res. 12 (2000) 47-51.

[4] F.I. Tsuji, E. Hill, Repetitive Cycles of Bioluminescence and Spawning in the Polychaete, Odontosyllis phosphorea, Biol. Bull. 165 (1983) 444-449.

[5] Y. Haneda, Luminous Swimming Polychaeta from the Banda Islands, Science Report of the Yokosuka City Museum. (1971) 34-35.

[6] E.N. Harvey, Bioluminescence, Academic Press, New York, 1952.

[7] T. Goto, Y. Kishi, Luciferins, bioluminescent substances, Angew. Chem. Int. Ed Engl. 7 (1968) 407-414.

[8] O. Shimomura, F.H. Johnson, Y. Saiga, Partial purification and properties of the Odontosyllis luminescence system, J. Cell. Comp. Physiol. 61 (1963) 275-292.

[9] O. Shimomura, J.R. Beers, F.H. Johnson, The cyanide activation of Odontosyllis luminescence, J. Cell. Comp. Physiol. 64 (1964) 15-21.

[10] G.L. Trainor, Studies on the Odontosyllis bioluminescence system, PhD, Harvard University, 1979.

[11] D.D. Deheyn, M.I. Latz, Internal and secreted bioluminescence of the marine polychaete Odontosyllis phosphorea (Syllidae), Invertebr. Biol. 128 (2009) 31-45.

[12] A. Verdes, D.F. Gruber, Glowing Worms: Biological, Chemical, and Functional Diversity of Bioluminescent Annelids, Integr. Comp. Biol. (2017).

https://academic.oup.com/icb/article/doi/10.1093/icb/icx017/3861033/Glowing-Worms -Biological-Chemical-and-Functional.

[13] N. Horii, Observation on luminous polychaeta, Odontosyllis undecimdonta from Toyama Bay, Japan Sea, Science Report of the Yokosuka City Museum. (1982) 1-3.

[14] O. Shimomura, Bioluminescence: Chemical Principles and Methods, World Scientific, 2006.

[15] S. Inoue, K. Okada, H. Tanino, H. Kakoi, A new hexagonal cyclic enol phosphate of 6 - $\beta$-hydroxypropionyllumazines from the marine swimming polychaete, Odontosyllis undecimdonta, Heterocycles. (1993).

[16] M.G. Grabherr, B.J. Haas, M. Yassour, J.Z. Levin, D.A. Thompson, I. Amit, X. Adiconis, L. Fan, R. Raychowdhury, Q. Zeng, Z. Chen, E. Mauceli, N. Hacohen, A. Gnirke, N. Rhind, F. di Palma, B.W. Birren, C. Nusbaum, K. Lindblad-Toh, N. Friedman, A. Regev, Full-length transcriptome assembly from RNA-Seq data without a reference genome, Nat. Biotechnol. 29 (2011) 644-652.

[17] N.I. Weisenfeld, V. Kumar, P. Shah, D.M. Church, D.B. Jaffe, Direct determination of diploid genome sequences, Genome Res. 27 (2017) 757-767.

[18] U.K. Laemmli, Cleavage of structural proteins during the assembly of the head of bacteriophage T4, Nature. (1970). http://link.springer.com/article/10.1038/227680a0.

[19] A. Shevchenko, M. Wilm, O. Vorm, M. Mann, Mass spectrometric sequencing of proteins silver-stained polyacrylamide gels, Anal. Chem. 68 (1996) 850-858.

[20] A. Shevchenko, H. Tomas, J. Havlis, J.V. Olsen, M. Mann, In-gel digestion for mass spectrometric characterization of proteins and proteomes, Nat. Protoc. 1 (2006) 2856-2860. 
[21] E. Weber, C. Engler, R. Gruetzner, S. Werner, S. Marillonnet, A modular cloning system for standardized assembly of multigene constructs, PLoS One. 6 (2011) e16765.

[22] A.A. Kotlobay, M.A. Dubinnyi, K.V. Purtov, E.B. Guglya, N.S. Rodionova, V.N. Petushkov, Y.V. Bolt, V.S. Kublitski, Z.M. Kaskova, R. Ziganshin, Y.V. Nelyubina, P.V. Dorovatovskii, I.E. Eliseev, B.R. Branchini, G. Bourenkov, I.A. Ivanov, Y. Oba, I.V. Yampolsky, A.S. Tsarkova, Bioluminescence chemistry of fireworm Odontosyllis: structure elucidation of luciferin and its enzymatic and non-enzymatic oxidation products, In Review. (n.d.).

[23] S.R. Eddy, Profile hidden Markov models, Bioinformatics. 14 (1998) 755-763.

[24] R.D. Finn, J. Clements, S.R. Eddy, HMMER web server: interactive sequence similarity searching, Nucleic Acids Res. 39 (2011) W29-37.

[25] S.F. Altschul, T.L. Madden, A.A. Schäffer, J. Zhang, Z. Zhang, W. Miller, D.J. Lipman, Gapped BLAST and PSI-BLAST: a new generation of protein database search programs, Nucleic Acids Res. 25 (1997) 3389-3402.

[26] L. Käll, A. Krogh, E.L.L. Sonnhammer, Advantages of combined transmembrane topology and signal peptide prediction - the Phobius web server, Nucleic Acids Res. 35 (2007) W429-W432.

[27] T.N. Petersen, S. Brunak, G. von Heijne, H. Nielsen, SignalP 4.0: discriminating signal peptides from transmembrane regions, Nat. Methods. 8 (2011) 785-786.

[28] J. Yang, R. Yan, A. Roy, D. Xu, J. Poisson, Y. Zhang, The I-TASSER Suite: protein structure and function prediction, Nat. Methods. 12 (2015) 7-8.

[29] V.N. Petushkov, M.A. Dubinnyi, A.S. Tsarkova, et al., A novel type of luciferin from the Siberian luminous earthworm Fridericia heliota: structure elucidation by spectral studies and total synthesis, Angew. Chem. Int. Ed Engl. 53 (2014) 5566-5568. 


\section{Supplementary Material to:}

Luciferase of the Japanese syllid polychaete Odontosyllis umdecimdonta

\section{Authors:}

Darrin T. Schultz ${ }^{1 *}$, Alexey A. Kotlobay ${ }^{2 *}$, Rustam Ziganshin ${ }^{2}$, Artyom Bannikov ${ }^{2,3}$, Nadezhda M. Markina ${ }^{2}$, Tatiana V. Chepurnyh ${ }^{2}$, Ekaterina S. Shakhova ${ }^{2}$, Ksenia Palkina $^{2}$, Steven H.D. Haddock ${ }^{4,5}$, llia V. Yampolsky ${ }^{2,6, \#, ~ Y u i c h i ~ O b a ~}{ }^{7, \#}$

\section{Affilations:}

${ }^{1}$ Department of Biomolecular Engineering and Bioinformatics, University of California Santa Cruz, 1156 High Street, Santa Cruz, California 95064, United States

${ }^{2}$ Shemyakin-Ovchinnikov Institute of Bioorganic Chemistry of the Russian Academy of Sciences, Miklukho-Maklaya, 16/10, Moscow, Russian Federation 117997

${ }^{3}$ Planta LLC, Bolshoi Boulevard, 42 Str 1, Office 335; Moscow, Russia 121205

${ }^{4}$ Monterey Bay Aquarium Research Institute, 7700 Sandholdt Road, Moss Landing, California 95039, United States

${ }^{5}$ Department of Ecology and Evolutionary Biology, University of California Santa Cruz, 1156 High Street, Santa Cruz, California 95064, United States

${ }^{6}$ Pirogov Russian National Research Medical University, Ostrovitianova 1, Moscow 117997, Russia

${ }^{7}$ Department of Environmental Biology, Chubu University, Kasugai 487-8501, Japan

* - These authors contributed equally to this work.

\# - Corresponding author. Correspondence and requests for materials should be addressed to ivyamp@ibch.ru and yoba@isc.chubu.ac.jp 


\title{
Supplementary Methods
}

\author{
Genomic DNA isolation and sequencing
}

Genomic DNA Isolation

Genomic DNA of one O. undecimdonta specimen, OdonB, was isolated using the Omega Biotek E.Z.N.A. Mollusc DNA kit (product number D3373). A $30 \mathrm{mg}$ sample of RNAlater-preserved tissue yielded $24 \mu \mathrm{g}$ of DNA at $80 \mathrm{ng} / \mu \mathrm{l}$ in $300 \mu \mathrm{L}$. A $1 \mu \mathrm{l}$ sample of OdonB DNA was imaged on a $1 \%$ agarose gel in a $150 \mathrm{~V}$ field for 45 minutes and was found to be larger than the $10 \mathrm{kbp}$ ladder. We did not perform pulse field gel electrophoresis to image the size distribution of the DNA greater than $10 \mathrm{kbp}$.

Genome Library Prep

We prepared both a 10X Genomics chromium DNA library [1], as well as a PCR-free whole genome shotgun library. For the 10X Genomics chromium library preparation, we sent a sample of the DNA to the UC Davis DNA Technologies Core where they performed the library prep and a single lane of 2x150 PE sequencing on an Illumina HiSeq 4000.

To prepare a PCR-free whole genome shotgun library, we sheared $1.5 \mu \mathrm{g}$ of DNA in 50 $\mu \mathrm{l}$ of 1 xTE using a Bioruptor sonication device on setting $\mathrm{HIGH}$, for 30 seconds ON/30 seconds OFF for 13 cycles until the DNA size distribution mode was 350bp. Every five shearing cycles we removed the DNA tube from the Bioruptor, vortexed it, and quickly spun down the contents. We used $1 \mu \mathrm{g}$ of sheared DNA as input for the Illumina TruSeq DNA PCR-Free library prep kit. The final library concentration was $6.68 \mathrm{ng} / \mu \mathrm{L}$ and uses the TruSeq i7 index ACAGTG(A). This library was pooled and sent to the UC Davis DNA Technologies Core for 2x150 PE sequencing on an Illumina HiSeq 4000. The sequencing run produced $39,628,963$ read pairs.

\section{Genome Assembly}

To assemble the genome, we used the 10X Genomics Supernova assembler v1.1.2. We opted to not use the PCR-free shotgun data to assemble the genome due to low predicted coverage, approximately 16x assuming a conservative guess of a $700 \mathrm{Mbp}$ genome size. All computation was performed using Haddock lab computational resources at the Monterey Bay Aquarium Research Institute. We used the simple command "supernova run --id <runid > --fastqs <path to fastq $>$ / --localmem $=500$ " to 
assemble the genome using $500 \mathrm{~GB}$ of memory and 96 cores [1]. This took approximately three days to complete.

RNA sequencing protocol and transcriptome assembly

RNA Isolation

Total RNA intended for an Illumina RNA-seq library was isolated using the Trizol protocol on an RNAlater-preserved specimen (OdonA) from the same collection location, date, and time as sample OdonB. The final RNA yield from a $40 \mathrm{mg}$ OdonA Trizol extraction was $170 \mathrm{ng} / \mu \mathrm{l}$ quantified by nanodrop in $45 \mu \mathrm{l}$, or $7.65 \mu \mathrm{g}$.

We also isolated total RNA from another individual, OdonC, to use downstream for Oxford Nanopore cDNA full-length sequencing. We isolated total RNA from OdonC using the manufacturer's recommended protocol. OdonC also has the same collection parameters as OdonB and OdonA. The final RNA concentration from approximately 30 mg of tissue was $14.2 \mu \mathrm{g}$ from $142 \mathrm{ng} / \mu \mathrm{l}$ in $100 \mu \mathrm{l}$ of $\mathrm{ddd}_{2} \mathrm{O}$, quantified by qubit.

Illumina cDNA library prep and sequencing

A template-switching Illumina RNA-seq library from OdonB total RNA was prepared at Evrogen (Moscow, Russia) with a TruSeq Stranded mRNA Library Prep Kit v2 with the i7 index ACAGTG(A). The library was sequenced at the UC Davis DNA Technologies Core on an Illumina HiSeq $40002 \times 150$ PE run to a depth of 32,457,166 read pairs.

cDNA Synthesis for 2D ONT Sequencing

For cDNA sequencing on the Oxford Nanopore Technologies Minion, we first synthesized cDNA from sample OdonC. All primers used in the following protocol were adapted from [2]. To $50 \mathrm{ng}$ OdonC total RNA in $8 \mu \mathrm{l}$, we added $2 \mu \mathrm{l}$ of a modified SmartSeq2

Oligo

dT

primer

(5' - /5Me-isodC/AAGCAGTGGTATCAACGCAGAGTACTTTTTTTTTTTTTTTTTTTTTTTTT TTTTTVN-3') synthesized by IDT, $1 \mu \mathrm{L}$ of $10 \mathrm{mM}$ dNTPs. We mixed by vortexing and spun down briefly. We incubated this mixture at $65^{\circ} \mathrm{C}$ for five minutes and snap-cooled on a freezer block in ice. To this reaction we added $4 \mu \mathrm{l}$ of $5 \times \mathrm{RT}$ buffer, $1 \mu \mathrm{l}$ of $100 \mathrm{mM}$ DTT, $1 \mu \mathrm{l}$ of RNaseOUT, and $2 \mu \mathrm{l}$ of $10 \mathrm{mM}$ strand-switching oligo (5'-AAGCAGTGGTATCAACGCAGAGTACATrGrGrG-3'). This mixture was mixed by vortexing and spun down briefly, then incubated for 2 minutes at $42^{\circ} \mathrm{C}$ on a thermal cycler. Then, $1 \mu \mathrm{l}$ of SuperScript IV enzyme (200 U/ $\mathrm{Ll}$ ) was added to the mixture and mixed with five $1 \mu$ pipette strokes. The reverse transcription reaction was carried out 
as follows: 10 minutes at $50^{\circ} \mathrm{C}$ for $\mathrm{RT}$, then 10 minutes at $42^{\circ} \mathrm{C}$ for strand switching, then 10 minutes at $80^{\circ} \mathrm{C}$ for heat inactivation.

To amplify the cDNA, we set up three PCR reactions using the above RT reaction as input: $5 \mu \mathrm{l}$ of the RT reaction, $1.5 \mu \mathrm{l}$ of $10 \mathrm{mM}$ ISPCR primer (5'-AAGCAGTGGTATCAACGCAGAGT-3'), $18.5 \mu \mathrm{l}$ NFW, and $25 \mu$ of LongAmp Taq $2 x$ Master Mix. Reaction contents were mixed by gentle inversion then centrifuged to remove bubbles. The PCR reaction conditions were: one cycle of $95^{\circ} \mathrm{C}$ for 10 seconds, fifteen cycles of $95^{\circ} \mathrm{C}$ for 15 seconds then $64^{\circ} \mathrm{C}$ for 15 seconds then $65^{\circ} \mathrm{C}$ for 500 seconds, and one cycle of $65^{\circ} \mathrm{C}$ for 10 minutes.

The resulting cDNA was visualized on an agarose gel and all three amplicons were pooled together.

\section{Library Prep and 2D ONT sequencing}

From the pooled OdonC cDNA $1 \mu \mathrm{g}$ was used as input for the remainder of the standard SQK-LSK208 Oxford Nanopore Technologies 2D Strand switching cDNA sequencing protocol. The final library concentration after $2 \mathrm{D}$ adapter-ligated capture and prior to sequencing was $8.18 \mathrm{ng} / \mu \mathrm{l}$. The final library mass loaded to the flow cell was $98.16 \mathrm{ng}$ in $12 \mu \mathrm{l}$ of library. The flow cell used was a model FLO-MIN106, and the flowcell ID was FAF06207. We used MinKNOW v1.3.30 to control the sequencing run.

The sequencing run produced 428,172 fast5 read files. We used Albacore v1.1.1 to perform 2D basecalling on the reads and poretools v0.6.0 to extract reads from the basecalled fast5 files [3].

Transcriptome Assembly

Adapters were trimmed from the Illumina RNA-seq reads using SeqPrep2 [4]. We then de novo assembled a transcriptome using Trinity v2.1.1 with the option --SS_lib_type FR for read directionality and the --long_reads option using all 2D reads extracted from the Albacore-basecalled Oxford Nanopore reads [5].

\section{Read Mapping}

Illumina RNA-seq reads were mapped to the transcripts with bwa mem [6]. Oxford Nanopore Technologies cDNA reads were mapped to the transcripts with the splice-aware minimap2 [7]. Peptide matches were extracted from source transcripts, then the small sequences were mapped to the reference transcript with bwa aln [8]. This is the information that is displayed in Figure 2 of the main text. This procedure 
allows some amino acid mismatch when matching mass spectrometry hits to a DNA sequence. This provides the advantage of finding signal when population-level amino acid diversity is high.

\section{Mammalian cell culture}

HEK293NT were maintained in DMEM supplemented with $10 \%$ FBS and $1 \times$ Penicillin/Streptomycin ("fullDMEM") for all growth and passaging steps unless otherwise noted. For continuous culture, the cells were grown to approximately $70-80 \%$ confluency and then split 1:12 to be ready to be split again 3 days later.

To split cells from a $25 \mathrm{~cm}^{2}$ flask the culture medium was gently removed, $1 \mathrm{ml}$ PBS without $\mathrm{Mg} / \mathrm{Ca}$ was added to cover the surface of the cells. PBS was removed and 1 $\mathrm{ml} 0.025 \%$ Trypsin in $6 \mathrm{mM}$ EDTA was added to the side of the flask, not directly onto the cells. Solution was spread over the cells by gently "rocking" the flask several times. The flask was incubated at $37^{\circ} \mathrm{C}$ for 1-2 min. Then flask was rocked to completely dislodge the cells. After gently pipetting $80 \mu$ of cell suspension was transferred to the new flask supplied with $5 \mathrm{ml}$ of fullDMEM.

For preparing cells for transfection, $40 \mu \mathrm{l}$ of cell suspension described above was transferred to $2 \mathrm{~cm}$ cell culture dish supplied with $2 \mathrm{ml}$ of fullDMEM. After 24 hours incubation at $37^{\circ} \mathrm{C}$ with $5 \% \quad \mathrm{CO}_{2}$ cells were transfected with FuGene 6 reagent (Promega, Fitchburg, WI, USA) in accordance to the manufacturer's protocol.

\section{Molecular cloning}

All cloning was performed by Golden Gate assembly. The synthesized genes were cloned into MoClo Level 0-SP vector from MoClo kit plasmid plCH41258. Level 1 eukaryotic expression plasmids were assembled into MoClo kit plasmid plCH47742 as a backbone, and the following parts were cloned in Level 0 vectors: CMV promoter, luciferase candidate gene, stop-codon containing DNA part and SV40poyA terminator. Prokaryotic expression plasmids were assembled with pCOOFY plasmid (T7 promoter) as a backbone and luciferase candidate gene as a single insert. A vector containing GFP was used as a positive control for cloning and expression.

\section{Sequences for Molecular Cloning}

Below are the DNA sequences of the dsDNA transcripts ordered from Twist for cloning into MoClo Level 0-SP. 
>odontosylis luciferase candidate \#1 (short ORF from DN46871_c9_g1_i5), seq to order at Twist GAAGACaaaATGTGGACTATATGAATATTCATGGATATGCCCCTTTTTGCATGGAACGTAGTG TTgagGACTGGGTGAATGCTCGTTTCTGGACTCGTTGTAAGGTTCGTACTGATCGTAGTTTAGA ACTGGCACCTGAAGAATATGCCACCTACTTTTGTTATAAGGTGTTTCGTGTAcaagATCCTAAA ATtGCTTGTCCAGGAATGGATGTGATCCTTTCACCTAACAAACTGACTGTACAACAAATGATGC AGAATAAGGAAATCGTGGAGTTGTAGCAGATAAATCTGAGCAATGGTGGGTTGGAATTATGCG TGAAATTATGCATCTGTCTAAGGACTTGAATGGTGTTCGTCAATTCCATTATGGATGGATCATC AACACAGCTACACAAAAGAATGTGGTTCCTTTGTGGTCACGTTATcaagGACCTACTGTTCCAG TACGTCGTGACATGCCTCGTATCATTAATGCCATGTCTAATGGCGGAGGAAACATCACCTTGGG AGATATTCGTAATTTCCACTGCTCTGCTGATCCAGACAGTGTTGCTGTCATCTGCCCTGAGTTT GGTTTCTTGTCCTATtcacCCGCTGAAACTATCGTTATGGTTCCAGTAAATGGATTAATCCTGA TGGGAATGACACAATCTGCAGATGGAGTACCCTTCGTAAAATCTGCCCTGTTTGTTGAGGTGTA TAACTTGCAACAGtcaggtaaGTCTTC

>odontosylis luciferase candidate \#2 (long ORF from DN46871_c9_g1_i2), seq to order at Twist GAAGACaaaATAAGTTAGCACTGTTATTAAGTATTGGATGTTGCCTGGTTGCCGTGAACTTTG CTTTACGTGCTACTATCATTCGTtgCCTTCGTAAAACTCGTAGTTGGTCAGAAATTGATTGTAC ACCACATCAGGACAAGCTGTATGAGGACTTTGACCGTATCTGGGCCGGAGATTACCTGTCAGTA TTTGCTGAATGGTTAGATAATCCCATCCCCCCAGAGTGGTCTGAGCAACGTCTGGCCACATACT GCATTGAGCGTGAATGTCACACTAATCAAGCTATGGTTGACTATATGAATATCCATGGATATGC CCCTTTTGCATGGAACGTAGTGTTgagGACTGGGTGAATGCTCGTTTCTGGACTCGTTGTAAG GTTCGTACTGACCGTAGTTTAGAACTGGCACCTGAAGAATATGCCACCTACTTTTGTTATAAGG TGTTTCGTGTAcaaGATCCTAAAATTGTtgCCCCTCCATGGATGTGATCCTTTCACCTAACAA ACTGACTGTACAACAAATGATGCAAAATAAGGAAATTCGTGGAGTTGTAGCAGATAAATCTGAG CAATGGTGGGTTGGAATTATGCGTGAAATCATGCATCTGTCTAAGGACTTGAATGGTGTTCGTC AATTCCATTATGGATGGATCATTAACACAGCTACACAAAAGAATGTGGTTCCTTTGTGGTCACG TTATcaaGGACCTACTGTTCCAGTACGTCGTGACATGCCTCGTATCATTAATGCCATGTCTAAT GGCGGAGGAAACATCACCTTGGGAGATATTCGTAATTTCCACTGCtcCGCTGATCCAGACAGTG TTGCTGTCATCTGCCCTGAGTTTGGTTTCTTGTCCTATAGTCCtGCTGAAACTATTGTTATGGT TCTTGTAAATGGATTAATCCTGATGGGAATGACACAATCTGCAGATGGTGTACCCTTCGTAAAA TCTGCACTGTTTGCTGAGATGTATAACCTTCAACAGtcaggtaaGTCTTC

>odontosylis luciferase candidate \#3 (long ORF from DN46871_c9_g1_i3), seq to order at Twist GAAGACaaatGAAGTTAGCACTGTTATTATCTATTGGATGTTGCCTGGTTGCCGTGAACTTTG CTTTACGTGCTACTATCATCCGTtgCCTTCGAAAACTCGTAGTTGGTCAGAAATTGATTGTAC ACCACATCAGGACAAGCTGTATGAGGACTTTGACCGTATCTGGGCCGGAGATTACCTGTCAGTA TTTGCTGAATGGTTAGATAATCCCATCCCCCCAGAGTGGTCTGAGCAACGTCTGGCCACATACT GCATTGAGCGTGAATGTCACACTAATCAAGCTATGGTTGACTATATGAATATCCATGGATATGC 
CCCTTTTTGCATGGAACGTAGTGTTgagGACTGGGTGAATGCTCGTTTCTGGACTCGTTGTAAG GTTCGTACTGACCGTAGTTTAGAACTGGCACCTGAAGAATATGCCACCTACTTTTGTTATAAGG TGTTTCGTGTAcaaGATCCTAAAATGCTtgCCCCTCAATGGATGTGATCCTTTCACCTAACAA ACTGACTGTACAACAAATGATGCAAAATAAGGAAATCCGTGGAGTTGTAGAGGATCGTTCTGAG CAATGGTGGGTTGGACTGATGCGTGAAATTAGTCATCTGTCTAAGGACTTGAATGGTGTGAAAC AATTCCATTATGGATGGATCATCAACACAGCTACACAAAAGAATGTGGTTCCTTTGTGGTCACG TTATCAGGGTCCTACTATTCCAGTACGTCGTGACATGCCTCGTATCATTAATGCCATTTCTAAT GGAGGAGGAAACATCACCTTGGGAGATTTTCGTAATTTTGAATGCTCACCTGATCCAGATAGTG TTGCTCTGATCTGCCCTGAGTTTGGTTTCTTGTCCTATGATCCCCCTGAAACTATTGTAATGGT GCCAGTAAATGAATTAATCCTGATGGGAATGACACAATCTGCTGATGGAGTACCTTTGTTGAAG TCTGCCCTTTTAGCTGAGATTGATGTCATTTCCCAAGCTtcaggtaaGTCTTC

>odontosylis luciferase candidate \#4 (long ORF from DN46871_c9_g1_i6), seq to order at Twist GAAGACaaaATGAATTAGCACTGTTACTTTCTATTGGATGTTGCCTGGTTGCCGTGAACTTTG CTTTACGTGCTACTATCATTCGTtgCCTCGTAAAACTCGTAGTTGGTCAGAAATCGATTGTAC ACCACATCAGGACaaaCTTTATGAGGACTTTGACCGTATCTGGGCCGGAGATTACCTGTCAGTA TTTGCTGAATGGTTAGATAATCCCATCCCCCCAGAGTGGTCTGAGCAACGTCTGGCCACATACT GCATTGAGCGTGAATGTCACACTAATCAAGCTATGGTTGACTATATGAATATCCATGGATATGC CCCTTTTTGCATGGAACGTAGTGTTgagGACTGGGTGAATGCTCGTTTCTGGACTCGTTGTAAG GTTCGTACTGACCGTAGTTTAGAACTGGCACCTGAAGAATATGCCACCTACTTTTGTTATAAGG TGTTTCGTGTAcaaGATCCTAAAATCGCTtgCCCCTCCATGGATGTGATCCTTTCACCTAACAA ACTGACTGTACAACAAATGATGCAAAATAAGGAAATTCGTGGAGTTGTAGAGGATCGTTCTGAG CAATGGTGGGTTGGATTGATGCGTGAAATCTCCCATCTGTCTAAGGACTTGAATGGTGTGAAAC AATTCCATTATGGATGGATCATCAACACAGCTACACAAAAGAATGTGGTTCCTTTGTGGTCACG TTATCAGGGTCCTACTATTCCAGTACGTCGTGACATGCCTCGTATCATTAATGCCATGTCTAAT GGCCGTGGAAACATCACCTTGGGAGATATTCGTAATTTCCACTGCtctGCTGATCCAGACAGTG TTGCTGTCATCTGCCCTGAGTTTGGTTTCTTGTCCTATTCACCCGCTGAAACTATCGTTATGGT TCCAGTAAATGGATTAATCCTGATGGGAATGACACAATCTGCAGATGGAGTACCCTTCGTAAAA TCTGCCCTTTTGTTGAgGTGTATAACCTGCAACAGtcaggtaaGTCTTC t

>odontosylis luciferase candidate \#4 (long ORF from DN46871_c9_g1_i6)

ATGAAGTTAGCACTGTTACTTTCTATTGGATGTTGCCTGGTTGCCGTGAACTTTGCTTTACGTG CTACTATCATTCGTtgCCTTCGTAAAACTCGTAGTTGGTCAGAAATCGATTGTACACCACATCA 
GGACaaaCTTTATGAGGACTTTGACCGTATCTGGGCCGGAGATTACCTGTCAGTATTTGCTGAA TGGTTAGATAATCCCATCCCCCCAGAGTGGTCTGAGCAACGTCTGGCCACATACTGCATTGAGC GTGAATGTCACACTAATCAAGCTATGGTTGACTATATGAATATCCATGGATATGCCCCTTTTTG CATGGAACGTAGTGTTgagGACTGGGTGAATGCTCGTTTCTGGACTCGTTGTAAGGTTCGTACT GACCGTAGTTTAGAACTGGCACCTGAAGAATATGCCACCTACTTTTGTTATAAGGTGTTTCGTG TAcaagATCCTAAAATCGCTtgCCCCTCCATGGATGTGATCCTTTCACCTAACAAACTGACTGT ACAACAAATGATGCAAAATAAGGAAATTCGTGGAGTTGTAGAGGATCGTTCTGAGCAATGGTGG GTTGGATTGATGCGTGAAATCTCCCATCTGTCTAAGGACTTGAATGGTGTGAAACAATTCCATT ATGGATGGATCATCAACACAGCTACACAAAAGAATGTGGTTCCTTTGTGGTCACGTTATCAGGG TCCTACTATTCCAGTACGTCGTGACATGCCTCGTATCATTAATGCCATGTCTAATGGCCGTGGA AACATCACCTTGGGAGATATTCGTAATTCCACTGCtctGCTGATCCAGACAGTGTTGCTGTCA TCTGCCCTGAGTTTGGTTTCTTGTCCTATTCACCCGCTGAAACTATCGTTATGGTTCCAGTAAA TGGATTAATCCTGATGGGAATGACACAATCTGCAGATGGAGTACCCTTCGTAAAATCTGCCCTT TTTGTTGAGGTGTATAACCTGCAACAGtcaggt

Methods for BLAST search for luciferase homolog search

Trinity to assemble transcriptomes from publicly available polychaete RNA-seq data of the following species: Amphinomidae (Pareurythoe californica SRR1926090 [9]), Chaetopteridae (Chaetopterus sp. SRR1646443 [10], Chaetopterus variopedatus SRR5590967, Mesochaetopterus minutus SRR1925760 [9], Phyllochaetopterus sp. SRR1257898 [11], Spiochaetopterus sp. SRR1224605 [11]), Eunicida (Eunice pennata SRR2040479, Eunice torquata SRR2005375 [9]), Cirratulidae (Cirratulus cirratus SRR5590966, Cirratulus spectabilis SRR3574861 [12]), Flabelligeridae (Flabelligera mundata SRR3574613 [12]), Acrocirridae (Macrochaeta clavicornis SRR1221445 [11]), Phyllodocidae (Phyllodoce medipapillata SRR2016923 [9]), Polynoidae (Harmothoe extenuata SRR1237766 [11], Harmothoe imbricata SRR2005364 [9] and SRR4841788 [13]), Syllidae (Syllis sp. SRR1224604 [11]), and Tomopteridae (Tomopteris helgolandica SRR1237767 [11]).

To search for luciferase homologs, we assembled transcriptomes of polychaetes using publicly available data. To do this, we downloaded the forward and reverse read fasta.gz files from the European Nucleotide Archive. The confirmed luminous species included in this analysis were Chaetopterus variopedatus [14], Harmothoe extenuata [15], Harmothoe imbricata [16], and Tomopteris helgolandica [17]. All other species mentioned above may be luminous, with the exception of: Eunice spp., Pareurythoe 
californica, Phyllodoce medipapillata [18]. Below is a list of species included in this analysis.

- Amphinomidae

○ Pareurythoe californica SRR1926090 [9]

- Chaetopteridae

- Chaetopterus sp. SRR1646443 [10]

- Chaetopterus variopedatus SRR5590967

- Mesochaetopterus minutus SRR1925760 [9]

- Phyllochaetopterus sp. SRR1257898 [11]

- Spiochaetopterus sp. SRR1224605 [11]

- Eunicida

- Eunice pennata SRR2040479

- Eunice torquata SRR2005375 [9]

- Cirratulidae

- Cirratulus cirratus SRR5590966

- Cirratulus spectabilis SRR3574861 [12]

- Flabelligeridae (Flabelligera mundata SRR3574613 [12]

- Acrocirridae

- Macrochaeta clavicornis SRR1221445 [11]

- Phyllodocidae

- Phyllodoce medipapillata SRR2016923 [9]

- Polynoidae

- Harmothoe extenuata SRR1237766 [11]

- Harmothoe imbricata SRR2005364 [9]

- Syllidae

- Harmothoe imbricata scale SRR4841788 [13]

- Syllis sp. SRR1224604 [11]

- Tomopteridae

○ Tomopteris helgolandica SRR1237767 [11]

Reads were trimmed using Trimmomatic paired-end version 0.35 with the options ILLUMINACLIP:TruSeq3-PE-2. fa:2:30:10 LEADING:3 TRAILING:3 SLIDINGWINDOW: $4: 15$ MINLEN:36 [19]. Trimmed reads were used to assemble each transcriptome using Trinity version 2.6.6 with default parameters [5]. The resulting transcriptome nucleotide fasta file was used in subsequent tblastn searches [20]. The nucleotide transcriptome was translated into longest complete ORFs using transdecoder version 5.3.0 [21]. This protein fasta file was used for blastp searches [20]. 
To search for homologs of the putative $O$. undecimdonta luciferase, we used the protein product of transcript c9g1i2 as a query against individual translated polychaete transcriptomes. For each blastp search, we limited the search to the top hit using -max_target_seqs 1 . The name of the top hit, percent identity of the blastp alignment, the length of the alignment, and the E-value of the blastp hit are reported in column 'c9g1i2 queried against transcriptomes' of table 1 (SI). To determine if the best blastp match in each polychaete transcriptome had a match to a known non-luciferase protein, the top blastp hit was used as a query in a blastp search against the $\mathrm{nr}$ database. The results of these blast searches are reported in the column "top hit from 'c9g1i2 queried against transcriptomes' queried against nr" of table 1 (SI).

To include the possibility that a homologous sequence was not translated by the Transdecoder software, we performed another search using the c9g1i2 protein sequence as a tblastn query against individual polychaete nucleotide transcriptomes. The standard translation table for each polychaete genome was used when performing the tblastn search $(-d b$ gencode 1$)$. As above, the blast results for this search are listed in the 'c9g1i2 queried against transcriptomes' column of table 1 (SI). The top tblastn hit was used as a blastx query against the $\mathrm{nr}$ database. The standard translation table was used for each query (-query_gencode 1). As above, the results of the blastx search are listed in column "top hit from 'c9g1i2 queried against transcriptomes' queried against $n r$ " of table 1 (SI). Blast searches with no results are listed as a blank line in table 1 (SI).

This script is available on github and is archived on zenodo [22]. 


\begin{tabular}{|c|c|c|c|c|c|c|c|c|c|c|}
\hline \multirow[b]{2}{*}{ Luminous } & \multirow[b]{2}{*}{ Species } & \multirow[b]{2}{*}{$\begin{array}{l}\text { search } \\
\text { type }\end{array}$} & \multicolumn{4}{|c|}{$\begin{array}{c}\text { c9g1i2 queried against } \\
\text { transcriptomes }\end{array}$} & \multicolumn{4}{|c|}{$\begin{array}{l}\text { top hit from 'c9g1i2 queried against } \\
\text { transcriptomes' queried against } \mathrm{nr}\end{array}$} \\
\hline & & & id of top hit in transcriptome & $\begin{array}{l}\text { c9g1i2 } \\
\% \\
\text { identity } \\
\text { to top } \\
\text { hit aln }\end{array}$ & $\begin{array}{l}\text { c9g1i2 } \\
\text { aln } \\
\text { length } \\
\text { with } \\
\text { top hit }\end{array}$ & $\begin{array}{l}\text { E-value } \\
\text { of top } \\
\text { hit }\end{array}$ & id of top hit in $\mathrm{nr}$ & $\begin{array}{l}\text { query } \\
\% \\
\text { identity } \\
\text { to top } \\
\text { nr hit }\end{array}$ & $\begin{array}{l}\text { query } \\
\text { aln } \\
\text { length } \\
\text { with } \\
\text { top hit }\end{array}$ & $\begin{array}{l}\text { E-value } \\
\text { of top hit }\end{array}$ \\
\hline Unknown & Chaetopterus sp. & blastp & TRINITY_DN22925_c0_g4_i2.p1 & 29.29 & 99 & 0.14 & gi|919024947|ref|XP_013396314.1| & 45.41 & 218 & $4.00 \mathrm{E}-56$ \\
\hline Yes & C. variopedatus & blastp & TRINITY_DN10776_c0_g1_11.p1 & 34.55 & 55 & 1.2 & gi|762122444|ref|XP_011444172.1| & 60.55 & 218 & 7.00E-95 \\
\hline Unknown & C. cirratus & blastp & TRINITY_DN39290_c0_g1_i2.p1 & 22.6 & 177 & 0.23 & gi|260828721|ref|XP_002609311.1| & 25.46 & 436 & 3.00E-17 \\
\hline Unknown & C. spectabilis & blastp & TRINITY_DN17418_c0_g6_i1.p1 & 28.57 & 56 & 0.38 & gi|762124899|ref|XP_011445465.1| & 31.95 & 169 & 1.00E-24 \\
\hline No & E. pennata & blastp & TRINITY_DN22511_c1_g3_i7.p1 & 24.71 & 85 & 1.9 & & & & \\
\hline No & E. torquata & blastp & TRINITY_DN35329_c0_g1_i1.p1 & 29.31 & 58 & 0.085 & gi|999972341|gb|KXJ11515.1| & 47.22 & 108 & $6.00 \mathrm{E}-24$ \\
\hline Unknown & F. mundata & blastp & TRINITY_DN40664_c0_g1_i21.p1 & 31.82 & 66 & 0.034 & gi|443726752|gb|ELU13811.1| & 63.64 & 110 & 4.00E-43 \\
\hline Yes & H. extenuata & blastp & TRINITY_DN20303_c1_g1_i1.p4 & 35.9 & 39 & 3.2 & gi|925170753|gb|ALC79271.1| & 50 & 102 & 3.00E-16 \\
\hline Yes & H. imbricata & blastp & TRINITY_DN88619_c0_g3_11.p2 & 23.81 & 63 & 0.42 & gi|585686475|ref|XP_006820332.1| & 22.76 & 123 & 0.002 \\
\hline Yes & H. imbricata scale & blastp & TRINITY_DN27258_c0_g1_i1.p1 & 23.66 & 93 & 0.56 & gi|675848026|ref|XP_009009573.1| & 25.2 & 127 & 2.00E-06 \\
\hline Unknown & M. clavicornis & blastp & TRINITY_DN1050_c0_g1_i12.p2 & 40 & 30 & 0.5 & gi|517142395|ref|WP_018331213.1| & 39.02 & 41 & 2 \\
\hline Unknown & M. minutus & blastp & TRINITY_DN31711_c6_g10_i2.p1 & 24.74 & 97 & 0.017 & gi|919079566|ref|XP_013420952.1| & 28.99 & 238 & $5.00 \mathrm{E}-20$ \\
\hline No & P. californica & blastp & TRINITY_DN143100_c2_g5_i2.p1 & 23.81 & 84 & 1.3 & gi|952549469|dbj|GAQ09912.1| & 26.41 & 337 & 8.00E-10 \\
\hline Unknown & Phyllochaetopterus sp. & blastp & TRINITY_DN32669_c0_g2_i3.p1 & 24.14 & 116 & 1.6 & gi|501451650|ref|WP_012475099.1| & 31.34 & 67 & 3.8 \\
\hline No & P. medipapillata & blastp & TRINITY_DN124544_c4_g1_i1.p1 & 22.22 & 81 & 3.8 & gi|443689881|gb|ELT92172.1| & 41.74 & 115 & 2.00E-18 \\
\hline Unknown & Spiochaetopterus sp. & blastp & TRINITY_DN6991_c0_g1_i1.p1 & 31.25 & 64 & 0.66 & gi|443710715|gb|ELU04831.1| & 90.55 & 127 & 4.00E-72 \\
\hline Unknown & Syllis $s p$. & blastp & TRINITY_DN3570_c0_g1_11.p1 & 25.68 & 74 & 1.2 & gi|936716028|ref|XP_014235942.1| & 32.81 & 64 & 9.1 \\
\hline Yes & T. helgolandica & blastp & TRINITY_DN9336_c0_g1_i1.p1 & 27.14 & 70 & 0.059 & gi|449687603|ref|XP_002155046.2| & 67.8 & 177 & 2.00E-88 \\
\hline Unknown & Chaetopterus sp. & tblastn & TRINITY_DN29231_c1_g6_i1 & 40 & 35 & 4.3 & gi|918306464|gb|KOF78510.1| & 86.5 & 163 & $5.00 \mathrm{E}-95$ \\
\hline Yes & C. variopedatus & tblastn & TRINITY_DN22098_c2_g2_i1 & 32.14 & 56 & 2.2 & gi|919092464|ref|XP_013383058.1| & 65.06 & 933 & \\
\hline Unknown & C. cirratus & tblastn & TRINITY_DN39290_c0_g1_i2 & 23.73 & 177 & 0.55 & gi|260828721|ref|XP_002609311.1| & 25.46 & 436 & $4.00 \mathrm{E}-17$ \\
\hline Unknown & C. spectabilis & tblastn & TRINITY_DN17418_c0_g6_i1 & 28.57 & 56 & 1 & gi|762124899|ref|XP_011445465.1| & 31.95 & 169 & 2.00E-24 \\
\hline No & E. pennata & tblastn & TRINITY_DN21730_c2_g1_i7 & 31.67 & 60 & 4.2 & gi|443696508|gb|ELT97202.1| & 65.19 & 563 & \\
\hline No & E. torquata & tblastn & TRINITY_DN35329_c0_g1_i1 & 29.31 & 58 & 0.39 & gi|999972341|gb|KXJ11515.1| & 47.22 & 108 & 8.00E-20 \\
\hline Unknown & F. mundata & tblastn & TRINITY_DN40664_c0_g1_i21 & 31.82 & 66 & 0.076 & gi|443726752|gb|ELU13811.1| & 63.64 & 110 & 1.00E-42 \\
\hline Yes & H. extenuata & tblastn & TRINITY_DN11376_c0_g1_i2 & 26.32 & 76 & 0.58 & & & & \\
\hline Yes & H. imbricata & tblastn & TRINITY_DN40076_c0_g1_i1 & 63.16 & 19 & 1.5 & gi|817108375|ref|WP_046481642.1| & 42.5 & 40 & 0.14 \\
\hline Yes & H. imbricata scale & tblastn & TRINITY_DN88619_c0_g3_i1 & 22.06 & 68 & 0.66 & gi|563607091|gb|AHB62358.1| & 40.83 & 120 & 7.00E-14 \\
\hline Unknown & M. clavicornis & tblastn & TRINITY_DN1718_c0_g1_i1 & 37.14 & 35 & 1.3 & gi|90081998|dbj|BAE90280.1| & 83.1 & 71 & 2.00E-34 \\
\hline Unknown & M. minutus & tblastn & TRINITY_DN28073_c0_g6_i2 & 33.33 & 60 & 2.9 & & & & \\
\hline No & P. californica & tblastn & TRINITY_DN146135_c1_g4_i6 & 32.73 & 55 & 2.5 & & & & \\
\hline Unknown & Phyllochaetopterus $s p$. & tblastn & TRINITY_DN32669_c0_g2_i1 & 24.14 & 116 & 3.3 & gi|973131399|gb|KUK83442.1| & 28.41 & 88 & 1.4 \\
\hline No & P. medipapillata & tblastn & TRINITY_DN125519_c1_g1_i1 & 44.83 & 29 & 3.9 & & & & \\
\hline Unknown & Spiochaetopterus sp. & tblastn & TRINITY_DN30856_c0_g1_i1 & 27.14 & 70 & 0.29 & gi|999982293|gb|KXJ21443.1| & 48.89 & 45 & 0.086 \\
\hline Unknown & Syllis sp. & tblastn & TRINITY_DN662_c0_g1_i1 & 37.14 & 35 & 0.73 & gi|918306464|gb|KOF78510.1| & 86.02 & 93 & $5.00 \mathrm{E}-49$ \\
\hline Yes & T. helgolandica & tblastn & TRINITY_DN14107_c0_g1_i1 & 28 & 75 & 1.2 & gi|919081493|ref|XP_013421813.1| & 48.57 & 70 & $1.00 \mathrm{E}-12$ \\
\hline
\end{tabular}

Table 1. (SI) Blast results from individual searches against polychaete transcriptomes. The top half of the figure shows results from using c9g1i2 as the blastp query against translated polychaete transcriptomes, the bottom half shows results of using c9g1i2 as a tblastn query against untranslated polychaete transcriptomes. All blast result cell colors are colored on a scale of red to white to blue, wherein red indicates a dissimilar blast result while blue indicates a similar blast result. The '\% identity to top hit aln' columns show the percent identity match for the blast alignments. The 'aln length with top hit' columns show the length of the blast matches. 


\section{Supplementary Results}

\section{ONT cDNA sequencing results}

With poretools we extracted the following quantities and types of reads: 421,040 forward reads, 343,752 2D reads, and 23,566 2D high quality reads. The total basecalled and extracted data yield was 328.4 million basepairs. The read length N50 was 836 basepairs. A hex-bin density plot and marginal density plot for the untrimmed reads are visualized in Figure 6.

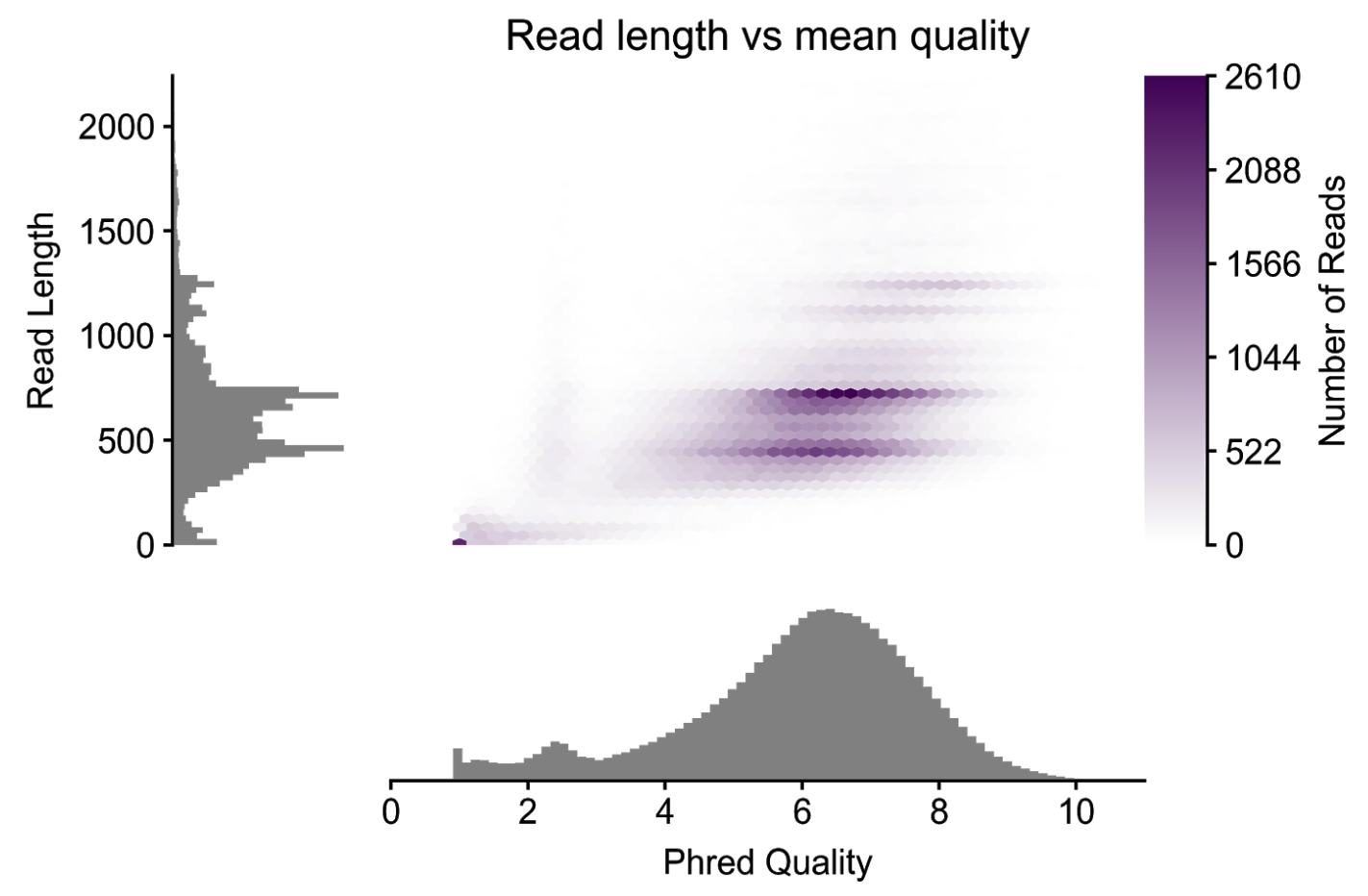

Fig. 5. (SI) A marginplot of the basecalled $2 \mathrm{D}$ reads' read lengths versus mean quality scores [23]. The average read quality is lower than what one would expect from a 2D sequencing run, however the read lengths indicate that many full-length transcripts were captured and sequenced as $2 \mathrm{D}$ reads.

\section{Genome Assembly Stats}

The 10X genome assembly resulted in 665,595 contigs contained in 555,643 scaffolds. The total contig and scaffold sequence sizes were $1.704 \mathrm{Gbp}$ and $1.741 \mathrm{Gbp}$. We believe that this total sequence size is approximately twice the nuclear genome size of around $850 \mathrm{Mbp}$ and that the 10X assembly has largely produced phased very small, phased haplotypes. 
The scaffold N50 was $4.1 \mathrm{kbp}$ and the L50 was 99,926 scaffolds. The contig N50 was $3.5 \mathrm{kbp}$ and the L50 was 125,775 contigs. There were 1,242 scaffolds greater than 50 $\mathrm{kbp}$ that comprised $5.5 \%$ of the genome. There were 189 scaffolds greater than 100 $\mathrm{kbp}$ in length, and 2 scaffolds greater than $250 \mathrm{kbp}$ in length.

Overall, this genome would benefit from a long read sequencing technology or another scaffolding technique to increase the N50. Future research plans include determining if the 10X assembly is comprised of phased haplotypes or if it is a pseudohaploid representation of the genome. Due to budgetary constraints, we plan to scaffold the genespace of this genome using RNAseq reads with RAS

\section{Transcriptome Assembly Stats}

The hybrid transcriptome assembly resulted in 256,027 unique transcripts in 142.1 Mbp. The transcript N50 is $737 \mathrm{bp}$. There are 30,599 transcripts greater than $1 \mathrm{~kb}$ in length, 5,331 transcripts greater than $2.5 \mathrm{~kb}$ in length, 689 transcripts greater $5 \mathrm{~kb}$, and 47 transcripts longer than $10 \mathrm{~kb}$.

This transcriptome contains more transcripts than is likely to be biologically relevant, but many of these are likely truncated transcripts from poor input RNA quality from using RNAlater.

\section{Peptide mass spectrometry matches}

The protein products of all four putative $O$. undecimdonta luciferase transcripts are similar in structure and sequence, except that c9g1i5 lacks $93 \mathrm{~N}$-terminal amino acids. See Figure 7 for a protein alignment and sites where individual peptide peaks were matched to transcripts by the Mascot software. 


\section{Homologous protein search}

The three highest percent identity blastp hits were only 30 amino acids at $40 \%$ similarity with and E-value of 0.5 in the Macrochaeta clavicornis transcriptome, 39 amino acids at $35.9 \%$ similarity with an E-value of 3.2 in the Harmothoe extenuata transcriptome, and 55 amino acids at $34.55 \%$ identity with an E-value of 1.2 in the Chaetopterus variopedatus transcriptome. When these top three hits were used as queries in a blastp search against the NCBI nr database, they had similar or higher percent identities to existing non-luciferase proteins $(39.02 \%, 50 \%, 60.55 \%)$, longer matching regions (41 aa, 50 aa, 218 aa), and varying E-values (2, 3E-16, 7.0E-95). These results generalize to all other blast searches that we conducted. Taken together, these results indicate that there are no conserved proteins in the assembled polychaete transcriptomes given that any blast hit better matches a sequence in the $\mathrm{nr}$ database than the protein of transcript c9g1i2.

\section{References}

[1] N.I. Weisenfeld, V. Kumar, P. Shah, D.M. Church, D.B. Jaffe, Direct determination of diploid genome sequences, Genome Res. 27 (2017) 757-767.

[2] S. Picelli, O.R. Faridani, Å.K. Björklund, G. Winberg, S. Sagasser, R. Sandberg, Full-length RNA-seq from single cells using Smart-seq2, Nat. Protoc. 9 (2014) 171-181.

[3] N.J. Loman, A.R. Quinlan, Poretools: a toolkit for analyzing nanopore sequence data, Bioinformatics. 30 (2014) 3399-3401.

[4] J. St. John, J. Eizenga, SeqPrep2, github, Santa Cruz, 2016. https://github.com/jeizenga/SeqPrep2.

[5] M.G. Grabherr, B.J. Haas, M. Yassour, J.Z. Levin, D.A. Thompson, I. Amit, X. Adiconis, L. Fan, R. Raychowdhury, Q. Zeng, Z. Chen, E. Mauceli, N. Hacohen, A. Gnirke, N. Rhind, F. di Palma, B.W. Birren, C. Nusbaum, K. Lindblad-Toh, N. Friedman, A. Regev, Full-length transcriptome assembly from RNA-Seq data without a reference genome, Nat. Biotechnol. 29 (2011) 644-652.

[6] H. Li, Aligning sequence reads, clone sequences and assembly contigs with BWA-MEM, arXiv. (2013). http://arxiv.org/abs/1303.3997.

[7] H. Li, Minimap2: pairwise alignment for nucleotide sequences, arXiv. (2017). http://arxiv.org/abs/1708.01492.

[8] H. Li, R. Durbin, Fast and accurate short read alignment with Burrows-Wheeler transform, Bioinformatics. 25 (2009) 1754-1760.

[9] S.C.S. Andrade, M. Novo, G.Y. Kawauchi, K. Worsaae, F. Pleijel, G. Giribet, G.W. Rouse, Articulating "Archiannelids": Phylogenomics and Annelid Relationships, with Emphasis on Meiofaunal Taxa, Mol. Biol. Evol. 32 (2015) 2860-2875.

[10] S. Lemer, G.Y. Kawauchi, S.C.S. Andrade, V.L. González, M.J. Boyle, G. Giribet, Re-evaluating the phylogeny of Sipuncula through transcriptomics, Mol. Phylogenet. Evol. 83 (2015) 174-183.

[11] A. Weigert, C. Helm, M. Meyer, B. Nickel, D. Arendt, B. Hausdorf, S.R. Santos, K.M. Halanych, G. Purschke, C. Bleidorn, T.H. Struck, Illuminating the base of the annelid tree 
using transcriptomics, Mol. Biol. Evol. 31 (2014) 1391-1401.

[12] Y. Li, K.M. Kocot, N.V. Whelan, S.R. Santos, D.S. Waits, D.J. Thornhill, K.M. Halanych, Phylogenomics of tubeworms (Siboglinidae, Annelida) and comparative performance of different reconstruction methods, Zool. Scr. 46 (2017) 200-213.

[13] W.R. Francis, L.M. Christianson, R. Kiko, M.L. Powers, N.C. Shaner, S.H. D Haddock, A comparison across non-model animals suggests an optimal sequencing depth for de novo transcriptome assembly, BMC Genomics. 14 (2013) 167.

[14] O. Shimomura, Bioluminescence: Chemical Principles and Methods, World Scientific, 2006.

[15] J.M. Bassot, M.T. Nicolas, Similar paracrystals of endoplasmic reticulum in the photoemitters and the photoreceptors of scale-worms, Experientia. 34 (1978) 726-728.

[16] M.-J. Miron, L. LaRivière, J.-M. Bassot, M. Anctil, Immunohistochemical and radioautographic evidence of monoamine-containing cells in bioluminescent elytra of the scale-worm Harmothoe imbricata (Polychaeta), Cell Tissue Res. 249 (1987) 547-556.

[17] A. Gouveneaux, J. Mallefet, Physiological control of bioluminescence in a deep-sea planktonic worm, Tomopteris helgolandica, J. Exp. Biol. 216 (2013) 4285-4289.

[18] P.J. Herring, Systematic distribution of bioluminescence in living organisms, J. Biolumin. Chemilumin. 1 (1987) 147-163.

[19] A.M. Bolger, M. Lohse, B. Usadel, Trimmomatic: a flexible trimmer for Illumina sequence data, Bioinformatics. (2014).

[20] S.F. Altschul, T.L. Madden, A.A. Schäffer, J. Zhang, Z. Zhang, W. Miller, D.J. Lipman, Gapped BLAST and PSI-BLAST: a new generation of protein database search programs, Nucleic Acids Res. 25 (1997) 3389-3402.

[21] B. Haas, A. Papanicolaou, Transdecoder (Find Coding Regions Within Transcripts), Github, n.d. https://github.com/TransDecoder/TransDecoder (accessed May 17, 2018).

[22] D.T. Schultz, Odontosyllis undecimdonta luciferase github repository, 2018. doi:10.5281/zenodo.124999.

[23] W. De Coster, S. D'Hert, D.T. Schultz, M. Cruts, C. Van Broeckhoven, NanoPack: visualizing and processing long read sequencing data, Bioinformatics. (2018). 